\title{
RELATION OF COLLAGEN FIBER ORIENTATION TO SOME MECHANICAL PROPERTIES OF HUMAN CORTICAL BONE*
}

\author{
F. GAYNOR EVANS \\ Department of Anatomy (Medical School) and Biomechanics Department (Highway Safety Research \\ Institute). The University of Michigan. Ann Arbor, Mich. 48104. U.S.A. \\ and \\ RAÚL VINCENTELLI \\ Facultad de Odontología. Cátedra de Histología y Embriología. Universidad Central de Venezuela. Caracas. \\ Venezuela
}

\begin{abstract}
The percentage of dark. light, and intermediate osteons and their fragments, as seen in polarized light, was determined for 53 cross-sections of embalmed, adult human bone. Dark osteons have collagen fibers predominantly parallel with the long axis of the osteon while in light osteons the fibers are at a sharp angle to the long axis of the osteon. Intermediate osteons are mixed.

Significant positive correlations were found between dark osteons alone, as well as when combined with their fragments, and the single shearing strength. Intermediate osteons alone, and when combined with their fragments, showed significant positive correlations with the (1) single shearing strength. (2) modulus of elasticity, and (3) percent elongation.

Significant negative correjations were found between light osteons alone. and when combined with their fragments, and the (1) single shearing strength and (2) modulus of elasticity.

No other significant correlations were found among the various types of osteon, alone, or when combined with their fragments, and the mechanical properties of the specimens.
\end{abstract}

\section{INTRODUCTION}

BONE is a heterogeneous, viscoelastic, anisotropic material whose mechanical properties, especially for animals, have been investigated rather extensively. However, there are relatively few studies on the relations between the mechanical properties of bone and its microscopic structure.

Probably the first person to suggest that collagen fibers play a mechanical role in bone was Gebhardt (1905) who studied the orientation of collagen fibers in osteons and made mechanical models to illustrate his concepts of their mode of action. He did not, however, experimentally determine any of the mechanical properties of bone.

The relation between the breaking load under bending and collagen fiber orientation in little pieces of compact bone cut parallel, radially, and tangentially to the long axis of ox tibias was investigated by Maj and Toajari (1937). From histological examination of sections of the specimens in ordinary and in polarized light, differences in the magnitude of the breaking load were explained on the basis of the predominant direction of the collagen fibers in the specimens. In the longitudinal specimens, which were the strongest, the collagen fibers were predominantly parallel with the long axis of the specimens but in the radial specimens, which were the weakest, only a few fibers were parallel with the major axis of the specimens. From their studies Maj and Toajari concluded that (1) the bending strength of compact bone is directly proportional to the number of collagen fibers in the plane of the section; (2) the cohesiveness of interfibrillar calcified substance

\footnotetext{
*Received 9 September 1968.
} 
is at least 6 times less than that of collagen fibers; (3) mechanical anisotropism of bone is dependent upon the distribution and direction of collagen fibers; and (4) the interfibrillar substance probably confers homogeneity and isotropic properties to compact bone.

From a study of cross-sections of bone in polarized light Olivo (1937) reported a constant parallelism between collagen fiber orientation and mechanical properties of compact bone from animals. In regions with a marked resistance to bending fracture, and also a high modulus of elasticity, most of the osteons were composed of vertical or steeply spiraling collagen fibers while in areas with a low fracture resistance the osteons had fibers more circularly or obliquely oriented.

Toajari (1938), on the basis of 271 observations, likewise concluded that the modulus of elasticity and the bending strength of bone are dependent upon collagen fiber orientation.

Recently the relation between collagen fiber orientation in individual osteons, as seen in polarized light, and their tensile strength and strain characteristics was investigated by Ascenzi and Bonucci $(1964,1967)$, who reported that in osteons with a marked longitudinal arrangement of bundles of fibers in successive lamellae, the ultimate tensile strength and modulus of elasticity seemed greater and the percent elongation under tension seemed lower than in osteons whose bundles in successive lamellae changed through an angle of about $90^{\circ}$.

In a later study (Ascenzi and Bonucci, 1968) they classified osteons as dark, light, or intermediate on the basis of their appearance in cross-sections of bone examined in polarized light. The dark osteons had collagen fiber bundles predominantly parallel with the long axis of the osteon whereas the light osteons consisted of collagen fiber bundles more nearly in the plane of the section. The intermediate osteons had both types of collagen fiber bundle arrangements. The dark osteons were stronger in tension and weaker in compression while the light osteons were just the reverse. The intermediate osteons were likewise intermediate in their strength characteristics.

Amprino (1958) found some relation between osteon fibers and the microhardness of bone. The microhardness was higher where the collagen fibers were perpendicular to and lower where they were parallel with the plane of the section. However, he believed this relationship was related more to the orientation of apatite crystals parallel with the fibers themselves.

The results of the studies by Ascenzi and Bonucci (1968) on individual osteons of different types led us to investigate the relations between the percentage of the break area formed by each type of osteon and its remnants (fragments) and the ultimate tensile and shearing strength, the tensile strain (percent elongation), and the modulus of elasticity of human cortical bone.

\section{MATERIALS AND METHODS}

The material used in our study consisted of cross-sections from 16 femoral, 28 tibial, and 9 fibular specimens, machined to a standardized size and shape, from the cortex of bones of embalmed, dissecting room cadavers. The specimens analyzed were chosen at random. The tensile and shearing stress (strength), tensile strain (percent elongation), and modulus of elasticity of the specimens had been previously determined by Evans and Lebow $(1951,1952)$. In an attempt to reduce the number of variables, specimens from only 2 cadavers were analyzed for their histological structure.

A histological analysis was made of crosssections, averaging $70 \mu$ in thickness, taken as close as possible to the fracture site of the specimen. After grinding, the sections were decalcified in Decal solution* and mounted,

\footnotetext{
* Manufactured by Omega Chemical Corporation, New York.
} 


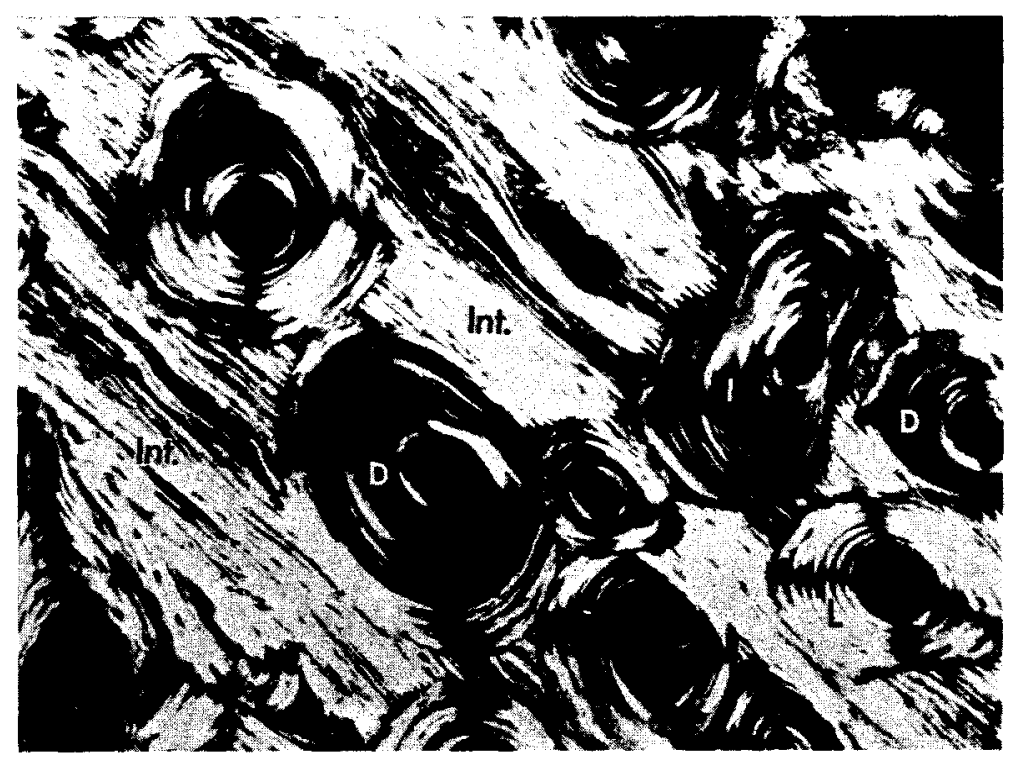

Fig. 1. A cross-section of embalmed human cortical bone, as seen in polarized light. $\mathrm{D}=$ dark osteon; I $=$ intermediate osteon; Int. = interstitial lamella; $\mathbf{L}=$ light osteon. 
unstained, in Kaiser's glycerol jelly for study in polarized light.

Photomicrographs of the sections between crossed polars were taken and prints, enlarged to a standardized size, were made on paper of a constant, known weight. An analysis of the different histological components in the sections, based on their appearance in polarized light, was made by the photomicrographicweight method used by Evans (1958) and Evans and Bang (1966, 1967). In order to minimize possible effects of technical variations during the analysis the original section was kept in place under the microscope so that it could be constantly examined. The method of hits was also tried but abandoned as not being suitable for our purpose.

When a decalcified section of bone is examined in polarized light (Fig. 1), the appearance of individual lamellae depends upon the orientation of their collagen fibers. On the basis of their appearance, osteons and their remnants were classified as dark, light, or intermediate (or mixed), as was done by Ascenzi and Bonucci (1968). Because our material consisted of cross-sections of specimens whose long axis was parallel with the shaft of the bone, we can infer that in dark osteons most of the collagen fibers are approximately perpendicular or at a steeply spiraling angle to the plane of the section. In light osteons most of the collagen fibers follow a circular or shallow, spiraling angle with respect to the plane of the section. The intermediate osteons have collagen fibers with both types of orientation.

The sections were analyzed for the percentage of the corrected break area formed by the different types of osteons and their remnants. The corrected break area represents the area of bone tissue in a section after all the major spaces (Haversian canals, Volkmann's canals, resorption spaces, etc.) have been removed. The region represented by the minor spaces (lacunae and canaliculi) is not accounted for by our method but it has an insignificant effect because, according to
Frost (1960), the combined area of the lacunae and the canaliculi in a vascular-free volume of human cortical bone (from long bones) is less than 3 per cent.

Coefficients of correlation between the percentage of the break area formed by the different types of osteons and the mechanical properties of the specimens, from which the sections were obtained, were calculated on computers. Analyses of variance and $t$-tests were also made to determine the significance of variations between histological components of sections from different bones.

\section{RESULTS}

During the histological analysis of our material it immediately became apparent that the different types of osteons and of osteon fragments did not appear in the same proportion in all sections examined.

The femoral sections had the highest and the tibial sections the lowest percentage of the break area formed by light osteons (Fig. 2). The percentage of dark osteons in the break area was considerably greater in the tibial than in either the femoral or the fibular sections which had approximately the same percentage of dark osteons. The fibular sections had the highest and the femoral sections the lowest percentage of the corrected break area formed by intermediate osteons although the differences among the femoral, tibial, and fibular sections were not as great as for the light and the dark osteons.

The highest percentage of light osteons plus fragments was found in the femoral

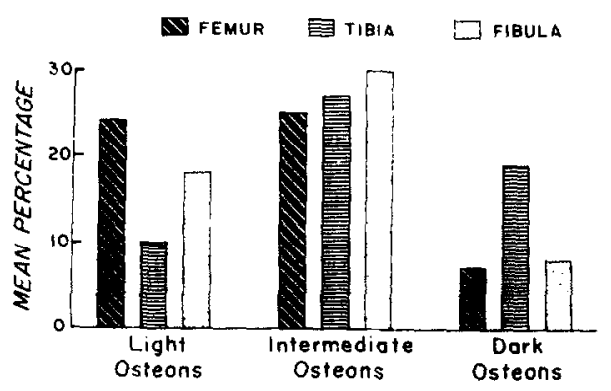

Fig. 2. Mean percentage of corrected break area formed by osteons. 
sections and the lowest in the tibial sections (Fig. 3). The percentage of dark osteons plus fragments was greatest in the tibial and least in the femoral sections, a condition almost identical with that for the dark osteons when considered alone. The intermediate osteons plus fragments formed the highest percentage of the corrected break area in the fibular sections and the lowest in the tibial sections; however, the difference between them was slight.

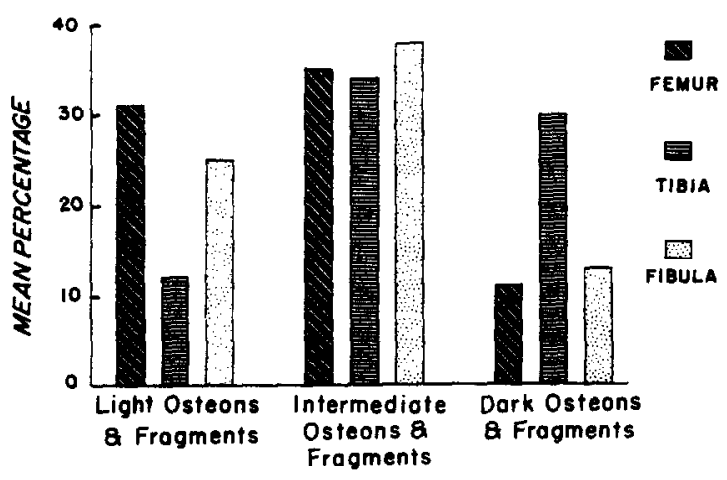

Fig. 3. Mean percentage of corrected break area formed by osteons and osteon fragments.

An analysis of variance and $t$-tests, made on computers, showed that the percentage of the corrected break area formed by dark osteons plus their fragments (Fig. 3) was significantly greater $(0.001$ level $)$ in the tibial than in either the femoral or the fibular sections. The femoral sections had a significantly greater $(0.001$ level) percentage of their corrected break area formed by light osteons plus fragments than did the tibial sections. The percentage of the corrected break area of the fibular sections formed by light osteons plus their fragments was significantly greater $(0.01$ level) than that of the tibial sections. No other significant differences were found between the means for the percentage of the break area of the sections formed by different types of osteons alone or by osteons plus their fragments.

The correlation coefficients (Table 1) revealed a very high positive correlation (at the 0.001 significance level) between the percentage of the corrected break area formed by dark osteons and the single shearing strength. A high positive correlation (at the 0.01 significance level) was found between the single shearing strength and the percentage of intermediate osteons in the corrected break area. Positive correlations were also found between the modulus of elasticity and the intermediate osteons (at the 0.02 significance level) and between the percentage elongation and the intermediate osteons (at the $\mathbf{0 . 0 5}$ significance level).

A very high negative correlation (at the 0.001 significance level) was seen between the single shearing strength and the percentage of light osteons in the corrected break area. The modulus of elasticity had a negative

Table 1. Correlation coefficients between the percentage of the corrected break area formed by different types of osteons and physical properties

\begin{tabular}{lcccc}
\hline & $\begin{array}{c}\text { Modulus of } \\
\text { elasticity }\end{array}$ & $\begin{array}{c}\text { Ultimate } \\
\text { tensile strength }\end{array}$ & $\begin{array}{c}\text { Percent } \\
\text { elongation }\end{array}$ & $\begin{array}{c}\text { Single } \\
\text { shearing strength }\end{array}$ \\
\hline $\begin{array}{l}\text { Light } \\
\text { osteons } \\
\text { Intermediate }\end{array}$ & $-0.315 \$$ & -0.052 & -0.085 & $-0.474^{*}$ \\
$\begin{array}{l}\text { osteons } \\
\text { Dark } \\
\text { osteons }\end{array}$ & $0.329 \ddagger$ & 0.283 & $0.313 \$$ & $0.408 \dagger$ \\
\hline
\end{tabular}

$$
\begin{aligned}
& *=0.001 \\
& \dagger=0.01 \\
& \ddagger=0.02 \\
& \S=0.05
\end{aligned}
$$


correlation (at the 0.05 significance level) with the light osteons.

No other significant correlations were found among the various types of osteons alone and the mechanical properties of the specimens.

Combining the various types of osteons and their fragments also revealed some interesting relations between histological structure and the mechanical properties of bone (Table 2). A very high positive correlation (at the 0.001 significance level) was found between the dark proportion of longitudinally oriented collagen fibers in bone and its bending strength. However, their methods of testing were rather simple and few quantitative data were given pertaining to the orientation of the collagen fibers and their specimens.

Recently Ascenzi and Bonucci (loc. cit.), using sophisticated techniques and measuring methods, demonstrated direct relationships between collagen fiber orientation in individual osteons and their tensile and compressive

Table 2. Correlation coefficients between the percentage of the corrected break area formed by different types of osteons plus fragments and physical properties

\begin{tabular}{lcccc}
\hline & $\begin{array}{c}\text { Modulus of } \\
\text { elasticity }\end{array}$ & $\begin{array}{c}\text { Ultimate } \\
\text { tensile strength }\end{array}$ & $\begin{array}{c}\text { Percent } \\
\text { elongation }\end{array}$ & $\begin{array}{c}\text { Single } \\
\text { shearing strength }\end{array}$ \\
\hline $\begin{array}{l}\text { Light } \\
\text { group }\end{array}$ & $-0.330 \dagger$ & -0.050 & -0.030 & $-0.475^{*}$ \\
$\begin{array}{l}\text { Intermediate } \\
\text { group } \\
\text { Dark } \\
\text { group }\end{array}$ & $0.302 \ddagger$ & 0.196 & $0.281 \neq$ & $0.319 \ddagger$ \\
\hline $\begin{array}{l}*=0.001 \\
+=0.02 \\
\ddagger=0.05\end{array}$ & 0.222 & 0.027 & -0.069 & $0.518^{*}$ \\
\hline$=0$ & & & & \\
\end{tabular}

osteons plus their fragments and the single shearing strength of the specimens. The intermediate osteons plus their fragments showed positive correlations (at the 0.05 significance level) with the (1) single shearing strength, (2) percent elongation, and (3) modulus of elasticity. The light osteons plus their fragments had a very high negative correlation (at the 0.001 significance level) with the single shearing strength and a high negative correlation (at the $\mathbf{0 . 0 2}$ significance level) with the modulus of elasticity.

No other significant correlations were found among the osteons plus their fragments and the mechanical properties of the specimens.

\section{DISCUSSION}

The investigations of Maj, Toajari, and Olivo, individually and collectively (loc. cit.), indicated a direct relationship between the stress-strain characteristics and their modulus of elasticity. Their research $(1964,1967)$ on the relation between tensile stress and strain and modulus of elasticity of individual osteons and the orientation of the collagen fibers led us to the present study in which we hoped to demonstrate similar relationships between the percentage of dark osteons in the crosssections of our specimens and some of the mechanical properties.

Our results did not correspond with our expectations. No significant relationships were found between the percentage of dark osteons, either alone or when combined with their fragments in the break area, and the ultimate tensile stress and strain, or the modulus of elasticity of the specimens. However, a very high positive correlation, at the 0.001 significance level, was found between the percentage of dark osteons in the break area of our specimens and the single shearing 
strength. The same was true for the combination of dark osteons and their fragments. A negative correlation, at the same significance level, was found between the light osteons alone, and when combined with their fragments, and the single shearing strength.

The absence of a positive correlation between dark osteons and the tensile stress and strain and the modulus of elasticity of our specimens may be due to the fact that the dark osteons form too small a proportion of the break area to have any significant effect. Thus, the greatest proportion of dark osteons alone (Fig. 2) in the break area was about 20 per cent while dark osteons plus their fragments (Fig. 3) constituted only 30 per cent of the break area.

Osteons are not simple tubular structures, as shown by Filogamo (1946), Koltze (1951), and Cohen and Harris (1958), but are structures that ramify quite extensively. The very high positive and negative correlations of the single shearing strength with the groups of dark osteons and fragments and of light osteons and fragments, respectively, may be explained partially by the orientation of the collagen fibers in the two groups of osteons with respect to the direction of the shearing force. The ultimate tensile strength, percent elongation (tensile strain), and modulus of elasticity were determined from tests in which the load was applied parallel with the long axis of the specimens. The shearing strength, however, was determined by applying the force perpendicular to the long axis of the specimen. Therefore, the predominant direction of the collagen fibers in the dark osteons would be perpendicular (or normal) to the direction of the shearing force whereas in the light osteons and their fragments the predominant direction would be more nearly parallel with the shearing force. Consequently, the light osteons would not resist shearing as well as the dark osteons because shearing occurs more easily in the direction of the collagen fibers than transverse to them. The collagen fibers in the dark osteons are in a better orientation to resist tension the shearing.

We are, of course, aware of the existence $i$ the literature of considerable controversy 0 the fine architecture of the osteon an consequently of the interpretation whicl should be given to its appearance in polarizer light. Our classification of osteons as light intermediate, or dark is the same as that user by Ascenzi and Bonucci (1968). Our inter pretation of the other collagen fibers is similar to that of Smith (1960) who, fror studies of human secondary osteons which were stained by Weidenreich's method, recognized three different types or patterns of collagen fiber orientation. The three patterns differed from one another in the relative numbers of longitudinal and circumferential fibers which they contained and in the degree of lamellation which they exhibited. Smith correlated the instance of the three fiber patterns with the relative ages of the regions of the bone in which they occurred.

By studying serial cross-sections of osteons in polarized light Filogamo (loc, cit.) demonstrated variation in the collagen fiber orientation along the axis of the osteon. Marked variation occurred in 20 per cent of his samples from old individuals but only very minor variations were found when the samples were taken from young individuals. Because our sections were not taken exactly at the fracture site differences in the collagen fiber orientation, as well as in the size and shape of the osteon cross-sections, unavoidably occurred. This introduced an uncontrollable variable which lowered our correlation figures.

The orientation of collagen fibers in the interstitial lamellae of our sections was not included in our analysis. Their appearance in polarized light in almost every case was that of regularly alternating dark and light lamellae somewhat similar to what we found in the light osteons. In most of our specimens the percentage of the corrected break area formed by the interstitial lamellae was rather low. Coefficients of correlation, limited to sections 
in which the interstitial lamellae formed less than 25 per cent of the corrected break area, did not produce consistently higher values while lowering their degree of significance because of reduction in sample size.

The possibility of determining the predominant orientation of the collagen fiber by scanning an entire cross-section, instead of determining the collagen fiber orientation for each individual osteon and osteon fragment, was also investigated. Photoelectric measurements from different sections, as seen in polarized light, were discarded because of technical variations in the section preparation which produced readings that were not directly comparable. The use of a photoelectric method, either averaging or of the spot type, would be very convenient for studies of this nature provided rigidly standardized technical procedures were adhered to during the preparation of the sections. This type of study has been planned for the future.

Collagen fiber orientation is, of course, only one of several structural variables that may affect the mechanical properties of bone.

As long ago as 1876 Rauber pointed out that the organization of bone substance into lamellae that form partial planes, partial curved plates, and rods all contribute to the strength of bone. Of particular importance is the use of curved plates to form hollow columns of circular or elliptical cross-sections, representing long bones in miniature, for the construction of long bones. These hollow columns (osteons or Haversian systems) are predominantly parallel with the long axis of the intact bone, corresponding to the mesh of the vascular network they contain. Furthermore, Rauber pointed out that the diameter of the Haversian canal as well as the thickness of its walls vary extensively with anatomical location within the bone and with age.

The effect of the orientation of the osteons is reflected by directional differences in the strength of bone, as demonstrated by Rauber (loc. cit.), Hülsen (1896), Dempster and Liddicoat (1952), Dempster and Coleman
(1961), and Evans (1964). All these investigators found that the strength of bone (both tensile and compressive) was greater in a direction parallel with the long axis of the of the bone, and hence the predominant direction of its osteons, than in a direction transverse to its long axis.

The percentage of the fracture area formed by osteons and their fragments and by interstitial lamellae, regardless of their collagen fiber orientation, is also known to influence the strength characteristics and other mechanical properties of bone.

Evans (1958) suggested that one of the reasons that compact human fibular bone has a greater tensile strength than similar femoral bone is that fibular bone is composed of a relatively small number of large osteons and osteon fragments while femoral bone consists of a large number of small osteons and osteon fragments. Thus, the larger the number of osteons and their fragements in a given area of bone, the lower its tensile strength.

Fractures tended to follow the cement lines, indicating that the cement substance was the weakest material in bone. Consequently, the larger the number of osteons in a given area of bone the greater the amount of cement substance where failure can occur. On the basis of a few specimens examined in polarized light, Evans suggested that a contributing factor to the greater tensile strength of the fibular specimens was their collagen fiber orientation, which was predominantly parallel with the long axis of the osteon and thus in a good orientation to resist tensile stress. This suggestion regarding the relation between collagen fiber orientation and tensile strength of bone was later confirmed by Ascenzi and Bonucci $(1966,1967)$.

Currey (1959) reported that the tensile strength of ox compact bone had a negative correlation with the amount of reconstruction and hence the number of Haversian systems or osteons in it. Two complementary explanations for his results were given: (1) immature Haversian systems have large central canals 
which reduce the amount of bone substance per unit area and (2) newly formed osteons are not as fully mineralized and are, therefore, presumably weaker than the surrounding primary bone. In a later publication Currey (1962) pointed out that the numerous small discontinuities in bone (lacunae, canaliculi, blood vessels, and muscle insertions) act as stress raisers. However, in mammalian bone the orientation of the lacunae and blood vessels reduces, but does not abolish, their stress concentrating effect in relation to dangerous stresses acting upon the bone.

An investigation of differences in the compressive and impact bending strength and microhardness of primary and secondary Haversian bone from cattle, a tortoise, rabbit, and man was recently made by Heřt et al. (1965). Primary bovine bone has a higher axial compressive strength than Haversian bone but no essential differences were found between the axial compressive strength of primary and secondary bone from a tortoise, rabbit, or man. In all tests the microhardness of primary bone was equal to or greater than that of secondary bone. They concluded that the mechanical properties of bone were not improved by remodeling, which increased the number of osteons and their fragments.

Recently Evans and Bang (1967) have shown a high positive correlation (significant at the 0.02 level) between the ultimate tensile strength of human compact bone and the percentage of the corrected break area formed by interstitial lamellae; between Rockwell hardness and the number of osteons $/ \mathrm{mm}^{2}$ (significant at the 0.01 level); and between hardness and the percentage of the corrected break area formed by osteons (significant at the 0.01 level).

They also found high negative correlations between the tensile modulus of elasticity and the percentage of the original break area formed by spaces (significant at the 0.01 level); between the ultimate tensile strength and the percentage of the corrected break area formed by osteons (significant at the 0.01 level); between the single shearing strength and the average area $\left(\mu^{2}\right)$ /osteon fragment or remnant (significant at the 0.02 level); and between hardness and the percentage of the original break area formed by spaces (significant at the 0.001 level). Negative correlations (significant at the 0.05 level) were found between the modulus of elasticity and the average area $\left(\mu^{2}\right) /$ osteon; and between the single shearing strength and the average area/osteon.

The mineral composition of bone as well as the distribution and orientation of the apatite crystals are other factors affecting the mechanical properties of bone.

The relative influence of all the above factors on the mechanical properties of bone is not known. However, a program of multiple correlation analysis and a study of the relations of the mineral distribution in bone, as revealed by microradiography, to its mechanical properties are in progress in our labora-

\section{SUMMARY AND CONCLUSIONS}

Cross-sections from 16 femoral, 28 tibial, and 9 fibular specimens, chosen at random, of human cortical bone were analyzed in polarized light with respect to the percentage of the break area formed by dark, light, and intermediate osteons and their fragments. Femoral sections had the highest and tibial sections the lowest percentage of light osteons plus fragments in their corrected break area. Dark osteons plus fragments were most abundant in the tibial and least in the femoral sections. This is also true for dark osteons when considered alone. Intermediate osteons plus fragments were most abundant in the fibular and least in the tibial sections. Intermediate osteons alone formed the highest percentage of the corrected break area in the fibular sections and the lowest in the femoral sections.

The differences between the means for the groups of light and dark osteons and of osteon fragments of the femoral and tibial sections and between the means for the groups of dark osteons and their fragments of the tibial and fibular sections were significant at the 
0.001 level. Differences between light osteons and their fragments in the tibial and fibular sections were significant at the 0.01 level. No other significant differences were found in sections from femoral, tibial, or fibular bone with respect to the histological structure.

A very high positive correlation (at the 0.001 significance level) was found between the percentage of the corrected break area formed by dark osteons and the single shearing strength. Intermediate osteons also had a very high positive correlation (at the 0.01 significance level) with the single shearing strength; (at the 0.02 significance level) with the modulus of elasticity; and (at the 0.05 signifiance level) with the percent elongation.

Light osteons had a very high negative correlation (at the 0.001 significance level) with the single shearing strength and (at the 0.05 signifiance level) with the modulus of elasticity.

No other significant correlations were found between the various types of osteons alone, or when combined with their fragments, and the mechanical properties of bone.

The significant correlations between the different types of osteons and the mechanical properties of human cortical bone are interpreted on the basis of the predominant direction of the collagen fibers in the osteons and their fragments.

Acknowledgement-This research was supported (in part) by Research Grant AM 03865-09 from the National Institutes of Health, U.S.P.H.S.

\section{REFERENCES}

Amprino, R. (1958) Investigations on some physical properties of bone tissue. Acta anat. 34, 161-186.

Ascenzi, A. and Bonucci, E. (1964) The ultimate tensile strength of single osteons. A cta anat. 58, 160-183.

Ascenzi, A. and Bonucci, E. (1967) The tensile properties of single osteons. Anat. Rec. 158, 375-386.

Ascenzi, A. and Bonnuci, E. (1968) The compressive properties of single osteons. Anat. Rec. 161, 377-392.

Cohen, J. and Harris, W. H. (1958) The three-dimensional anatomy of Haversian systems. J. Bone Jt. Surg. 40A, $419-434$.

Currey, J. D. (1959) Differences in the tensile strength of bone of different histological types. J. Anat. 93, 87-95.

Currey, J. D. (1962) Stress concentrations in bone. Q. Jlmicrosc. Sci. 103.111-133.
Dempster, W. T. and Coleman, R. F. (1961) Tensile strength of bone along and across the grain. $J$. appl. Physiol. 16, 355-360.

Dempster, W. T. and Liddicoat, R. T. (1952) Compact bone as a non-isotropic material. Am. J. Anat. 91. 331-362.

Evans, F. G. (1958) Relations between the microscopic structure and tensile strength of human bone. Acta anat. 35, 285-301.

Evans, F. G. (1964) Significant differences in the tensile strength of adult human compact bone. In Proc. of the First Eur. Bone und Tooth Symp. (Edited by $\mathrm{H}$. J. J. Blackwood), pp. 319-331. Pergamon Press, Oxford.

Evans, F. G. and Bang, S. (1966) Physical and histological differences between human fibular and femoral compact bone. In Studies on the Anatomy and Function of Bone and Joints. (Edited by F. G. Evans), pp. 142-155. Springer, Berlin.

Evans, F. G. and Bang, S. (1967) Differences and relationships between the physical properties and the microscopic structure of human femoral, tibial and fibular cortical bone. Am. J. Anat. 120, 79-88.

Evans, F. G. and Lebow, M. (1951) Regional differences in some of the physical properties of the human femur. J. appl. Physiol. 3, 563-572.

Evans, F. G. and Lebow, M. (1952) The strength of human compact bone as revealed by engineering technics. Am. J.Surg. 83. 326-331.

Filogamo, G. (1946) Precisazioni sulla disposizione e sull'orientamento delle fibre collagene degli osteoni, nell'uomo. Ric. Morfologia 22, 1-5.

Frost. H. (1960) Measurement of osteocyles per unit volume and volume components of osteocytes and canaliculae in man. Henry Ford Hosp. med. Bull. 8, 208-211.

Gebhardt, W. (1905) Über funktionell wichtige Anordnungsweisen der feineren und grösseren Bauelemente des Wirbeltierknochens. II. Spezieller Teil. Arch. Entw. Mech. 20, 187-322.

Heřt, J., Kucera, P., Vávra, M. and Volenik, V. (1965) Comparison of the mechanical properties of both the primary and Haversian bone tissue. Acta anat. 61, 412-433.

Hülsen, K. K. (1896) Specific gravity, resilience and strength of bone. Bull. biol. Lab., St. Petersburg, 1 , 7-37 (Russian text with German summary).

Koltze, H. (1951) Studie zur aüsseren Form der Osteone. Z. Anat. Entw Gesch. 115. 584-596.

Maj, G. and Toajari, E. (1937) Osservazioni sperimentali sul meccanismo di resistenza del tessuto osseo lamellare compatto alle azioni meccaniche. Chir. Org. Mov. 22, 541-557.

Olivo, O. M. (1937) Rispondenza della funzione meccanica varia degli osteonj con la loro diversa minuta architettura. Boll. Soc. ital. Biol. sper. 12, 400-401.

Rauber, A. A. (1876) Elasticität und Festigheit der Knochen. Engelmann, Leipzig.

Smith, J. W. (1960) The arrangement of collagen fibres in human secondary osteones. J. Bone Jt Surg. 42B, 588605 .

Toajari, E. (1938) Resistenza meccanica ed elasticità del tessuto osseo studiata in rapporto alla minuta struttura. Monit. Zool. Ital. 48. 148-154. 